\title{
A Forest Change Detection using auto Regressive Model-Based Kernel Fuzzy Clustering
}

\author{
Madhuri Mulik , V. Jayashree , P . N. Kulkarni
}

\begin{abstract}
Satellite images are used for applications related to the forest change detection, forest cover management, and so on as remote sensing provides the rich source of information for change detection. In this paper, the vegetation indices play a major role in extracting the useful information from the satellite images and the commonly employed indices. This paper analyzes the imagery data from the remote sensing satellites for detecting the changes in the forest over the year's 2007-2017 using the pixel-based Bhattacharya distance. The indices from the satellite images are fed to the automatic segmentation model using the proposed Kernel Fuzzy Auto regressive (KFAR) model, which is the modified Kernel Fuzzy C-Means (KFCM) Clustering algorithm with the Conditional Autoregressive Value at Risk (CAVIAR). The forest change detection using the pixel-based Bhattacharya distance follows the segmentation, and the experimentation reveals that the proposed method acquired the minimal MSE and maximal accuracy of 0.0581 and 0.9211 .
\end{abstract}

Keywords: Kernel Fuzzy C-Means Clustering, CAVIAR, vegetative index, Satellite data, Forest change detection

\section{INTRODUCTION}

Satellite data are employed extensively for research for the past few years and mainly, in forecasting weather, monitoring the environmental conditions globally, geological, and geographical applications. The applications of remote sensing in the areas of natural hazards and disasters are recently trending and play a major role in creating awareness among the people regarding the environment specially, regarding the climatic changes in the environment. It is significant to note that the climate changes and growth of population raises the pressure on the highlands of the world. The outcome of the pressure is diverse that includes the following: growth in urban areas, intensified agriculture, degradation of the forest areas, lack of biodiversity, increasing degradation of the healthy land, and soil erosion [8]. Operational systems to monitor and update the forest maps are essential for many applications, like carbon budgeting, forest management, and habitat monitoring [16, 17] [9]. Change detection of forest refers to the method of determining the change regarding the

Revised Manuscript Received on December 15, 2019

* Correspondence Author

Ms. Madhuri B. Mulik*, PHD Scholar, Department of E\&TC Engi., Sharad Institute of Technology College of Engineering, Yadrav (Ichalkaranji),India. Email: mulik_madhuri2002@yahoo.co.in

Dr. V. Jayashree, Professor (PG), Electronics Department DKTE's College of Textile and Engineering, Ichalakaranji, India.

Email: jayashreevaddin@gmail.com

Dr. P . N. Kulkarni, Professor \& Head, Electronics and Communication Engineering Department Bagalkot, India. Email: pxnxkulkarni@gmail.com state of the object or phenomenon through observing them at various instances [18]. The ultimate aim of change detection in remote sensing involves the identification of the location geographically, recognizing them, and quantifying the types of change and finally, analyzing the accuracy outcomes [19]. Remote sensing data assists the change detection as the change of the interested object specifies the spectral behavior, such as value of reflectance or local texture that is differentiated using the changes influenced through certain factors, like illumination angles and viewing angles, state of the atmospheric, and moisture of the soil [20] [10].

Traditional methods using the remote sensing data depend on the image-based vegetation indices, like Enhanced Vegetation Index, Soil-Adjusted Vegetation Index (SAVI), Normalized Difference Vegetation Index, and modified SAVI for accessing the condition of vegetation [11]. Additionally, there are few indices that reflect the spatial heterogeneity and vegetation patterns, like the landscape connectivity index [12] and Moving Standard Deviation (SD) index [13]. For the past few decades, there are a large number of spatial decision support system to support the decision makers in the areas of resource allocation and, particularly, spatial planning problems [14]. An idea for the automatic modification of geographical information system (GIS) databases using multispectral remote sensing data was used [15]. At present, object-based approaches are used for forest change detection for combining the contextual analysis for the visual interpretation with the quantitative aspect of pixel-based approaches [9].The primary intention of this research is to design and develop an approach for forest change detection using satellite images. This work proposes an enhanced forest change detection model based on pixel based method, which utilizes the Bhattacharya distance. Initially, two satellite images is considered from the same geographical area. Then, the pre-processing is applied on these images by the filtering approach. After the pre-processing stage, the feature extraction is performed based on the Vegetative Indices, such as RVI, NDVI, EVI, WDRVI, GARI, CI_Green, and GLAI. Here, the forest area segmentation is performed by the newly developed segmentation method, KFAR model, which is the integration of KFCM and auto regressive model. The segmented results from both the images are given to the proposed change detection model that utilizes the pixel based method with Bhattacharya distance. Thus, the proposed model generates the output effectively; detecting the changes in the satellite images. 


\section{LITERATURE REVIEW}

This section reviews eight existing methods of forest classification. The discussion follows: Manabu Watanabe et al. [1] developed the early detection method for detecting the deforestation using the L-band synthetic aperture radar (SAR) that was effective in detecting the later-stage of deforestation. The demerit of the method was that the HV image did not show appropriate change. Unmesh Khati et al. [2] developed a method for identifying the forest destruction using the SAR data that assisted the application of dual-pol SAR data to detect the forest cover. The drawback was that the method failed to perform poor with the Sentinel-1A/B data. Akansha Singh and Krishna Kant Singh [3] developed a method based on unsupervised detection based on the Kullback Leibler distance, which enabled better results in the occurrence of the natural disasters even under varied image acquisitions situations. The demerit of the method was that whenever the images were recorded from a single incidence angle that resulted in the information loss. Salman H. Khan et al. [4] established the forest change detection method based on Deep Neural Networks that accurately localized the change to predict the onset and offset timings. The method was not applicable in case of the large-scale annotated data set. Neha Gupta et al. [5] used an approach based on the binary descriptor to detect the change using the bitemporal optical satellite images that enabled the smoother visual outcomes. The drawback of the method was that it formed long codes to make the LBSP codes statistically weak. Hichem Sahbi [6] developed a method based on the relevance feedback and Context-Aware Canonical Correlation Analysis (CCA) that enhanced the performance of the change detection, but it was inadequate to analyze the structures. Oliver Cartus et al. [7] developed an error model to map the forest area that facilitated the frequent observation and timely detection, but the method was that it disregarded the backscatter contributions arising as a result of the interactions.

\section{EXPERIMENTATION}

\section{A. Forest change detection using the Kernel Fuzzy Auto regressive model based on the vegetative indices}

This paper utilizes the satellite images for the forest change detection and for the detection two satellite images covering the geographical area of the same region is chosen. Initially, the input satellite images are subjected to the pre-processing using the filtering method that makes the image fit for the further processing that includes the feature extraction and classification. From the pre-processed images, the vegetation indices are obtained that and subjected to the classification using the proposed clustering approach. The proposed approach is based on the KFAR model, which is modelled with the integration of the CAVIAR in the KFCM clustering approach. The clustering yields the forest and the non-forest segments from the satellite images, which are subjected to mapping based on the Bhattacharya distance. The pixel-based matching is employed for determining the changes in the pixels with respect to both the satellite images. Figure 1 shows the block diagram of forest change detection method. Let us consider two satellite images $A^{\prime}$ and $B^{\prime}$ of dimension $(P \times Q)$ in such a way that the image $A^{\prime}$ corresponds to the satellite image of instant $(t-1)$ and $B^{\prime}$ corresponds to the satellite image of the instant $(t-1)$. The satellite images of the same area coverage is chosen so that to detect the changes in the particular forest area within the duration $t$ and $(t-1)$.

\section{B. Preprocessing}

The major step is the pre-processing the input satellite images $A^{\prime}$ and $B^{\prime}$ of dimension $(P \times Q)$ in order ensure the effective processing in the further steps. For pre-processing, bilateral filter is applied, which is a spatially varying filter, aiming at preserving the edges when compared with the other filters. The bilateral filter finds the weighed sum of the neighbouring pixels, where the weight depends on the spatial and the intensity distance that assures the edge preservation. The pre-processed images are denoted as, $A$ and $B$.

\section{Extraction of the vegetative indices from the satellite image}

Vegetation index measures the measure of the greenness in the pixels of the input satellite image and the index defines the strength of the vegetation in the satellite image pixel-wise. In general, vegetation index [23] [25] [26] is the transformation of the spectral bands, which gives a clear view of the green plants found in the pixel in such a way that they are distinct in comparison with the other pixels of the input satellite image.

\section{Forest area segmentation using the proposed Kernel Fuzzy Autoregressive model:}

The proposed KFAR is the integration of the CAVIAR in the KFCM, which inherits the advantages of both KFCM [21] and auto regressive model, CAVIAR [22]. The advantage of CAVIAR is that it solves the following issues: It yields a model to estimate the required parameters with the known variables at time $(\tau-1)$, facilitates the estimation of the unknown parameters either through the optimization or any loss function, and enables the effective quality in estimation. On the other hand, the KFCM stands as the modification of the standard FCM with the kernel functions in such a way that the small deviations among the segments are managed effectively. The major role of the kernel functions is to map the input data of the non-linear type in the given dimensional area, which suffered from the complexity of dealing with the high-dimensional space, taking large time. Hence, the computational complexities are addressed using the integration of the CAVIAR in KFCM. Moreover, the segmentation using the standard KFCM did not consider the data of the previous predictions for enabling the current prediction and in addition, method failed in case of the additive noise. On the other hand, the non-linear mapping of the nodes as clusters is a simple and easy mechanism in KFCM. Thus, integrating CAVIAR with KFCM makes the segmentation process an effective, simple, and easy process through the consideration of the prediction results of the past in the current prediction. Following are the steps of the proposed algorithm,

Step 1: Initialization: The initialization is the first step that initializes the randomly chosen centroid as, $G_{k} ;(1 \leq k \geq M)$,

where, $M$ stands for the total centriods and let us assume the fuzzy c-partition as, $R$.

Published By:
Blue Eyes Intelligence Engineering
\& Sciences Publication


Consider the fuzzification parameter as, $u$, where $u$ varies between 1 and infinity and let us assume a factor $\omega$, which is greater than zero.

Step 2: Calculate the membership matrix: Let us consider the membership function $R=\left[U_{l k}\right]$ that is formulated as,

$$
U_{l k}=\frac{\left(\frac{1}{1-\kappa\left(z_{l}, G_{k}\right)}\right)^{\frac{1}{u-1}}}{\sum_{l=1}^{g}\left(\frac{1}{1-\kappa\left(z_{l}, G_{k}\right)}\right)^{\frac{1}{u-1}}}
$$

Where, $U_{l k}$ is the membership degree of each data point and the $k^{\text {th }}$ cluster center and $\kappa\left(z_{l}, G_{k}\right)$ refers to the kernel function that is calculated depending on the exponential kernel that is formulated as,

$$
\kappa\left(z_{l}, G_{k}\right)=\exp \left(\frac{-\sum_{l=1}^{g}\left|z_{k}^{a}-G_{k}^{a}\right|}{v^{2}}\right)
$$

The application of the exponential kernel functions is that it finds application in the real-world scenarios and the kernel function, $\kappa\left(z_{l}, G_{k}\right)$ computes the similarity among each data point $z_{l}$ with respect to the $k^{\text {th }}$ cluster center $G_{k}$. Whenever the data point $z_{l}$ rests away from the other data points, the kernel function $\kappa\left(z_{l}, G_{k}\right)$ remains small making the weighted sum of the data points remain highly robust. Thus, it is clear that the efficiency of segmenting the incomplete data is high using the proposed KFAR.

Step 3: Update the cluster centers: The cluster centers are updated following the update in the membership degree that is given as,

$$
G_{k}^{t+1}=\frac{\sum_{l=1}^{g} m_{l k}^{f} \kappa\left(z_{l}, G_{k}\right) \cdot z_{l}}{\sum_{l=1}^{g} m_{l k}^{f} \kappa\left(z_{l}, G_{k}\right)}
$$

Equation (3) is the standard equation of KFCM that is subjected to modification using the CAVIAR equation, for which the above equation is rearranged as follows,

$$
G_{k}^{t+1}-G_{k}^{t}=\frac{\sum_{l=1}^{g} m_{l k}^{f} \kappa\left(z_{l}, G_{k}\right) \cdot z_{l}}{\sum_{l=1}^{g} m_{l k}^{f} \kappa\left(z_{l}, G_{k}\right)}-G_{k}^{t}
$$

The CAVIAR update equation is given as,

$$
G_{k}^{t}(\alpha)=\alpha_{0}+\sum_{r=1}^{p} \alpha_{r} G_{k}^{t-r}(\alpha)+\sum_{i=1}^{q} \alpha_{i} \times L\left(z_{t-i}\right)
$$

Where, $\alpha_{0}$ symbolizes the co-vector of the unknown parameters and $c=p+q+1$ denotes the dimension of $\alpha$, and $L\left(z_{t-i}\right)$ signifies the fitness of $z_{t-i}$ that is computed based on the clustering distance between the centroid and the individual data point. The clustering distance is given as,

$$
L\left(z_{t-i}\right)=\frac{1}{M} \times \sum_{l=1}^{g} \sum_{k=1}^{M}\left\|z_{l}-G_{k}^{t-1}\right\|
$$

Where, $g$ represents the total data points in the cluster and $M$ corresponds to the total centroids. Now, let us assume that $p=q=2$ and hence, the equation (5) becomes,

$G_{k}^{t}(\alpha)=\alpha_{0}+\alpha_{1} G_{k}^{t-1}(\alpha)+\alpha_{2} G_{k}^{t-2}(\alpha)$

$+\alpha_{1} \times L\left(z_{t-1}\right)+\alpha_{2} \times L\left(z_{t-2}\right)$

The CAVIAR equation (7) is integrated in equation (4) as,

$$
\begin{aligned}
& G_{k}^{t+1}=G_{k}^{t}-\alpha_{1} G_{k}^{t-1}(\alpha)-\alpha_{2} G_{k}^{t-2}(\alpha)- \\
& {\left[\alpha_{0}+\alpha_{1} \times L\left(z_{t-1}\right)+\alpha_{2} \times L\left(z_{t-2}\right)\right]+\frac{\sum_{l=1}^{g} m_{l k}^{f} \kappa\left(z_{l}, G_{k}\right) \cdot z_{l}}{\sum_{l=1}^{g} m_{l k}^{f} \kappa\left(z_{l}, G_{k}\right)}}
\end{aligned}
$$

Where, $G_{k}^{t}, G_{k}^{t-1}(\alpha)$, and $G_{k}^{t-2}(\alpha)$ are the centroids of the previous iterations, and $L\left(z_{t-1}\right)$ and $L\left(z_{t-2}\right)$ are the fitness of the centroids in the iterations $(t-1)$ and $(t-2)$, respectively. It is clear from the above formula that the centroid of the new iteration is based on the centroids of the previous three iterations, $t,(t-1)$, and $(t-2)$, and their fitness measure, which is based on the clustering distance.

Step 4: Verify the stopping criterion: Upon the completion of the update process of the centroid and the membership degree, the stopping criterion is verified and the iteration is repeated from step 2 . The stopping criterion is given as,

$$
\boldsymbol{\vartheta}=\max \left|R^{t+1}-R^{t}\right|
$$

Whenever $\vartheta$ exceeds $\omega$, repeat from step 2 or else terminate. The results of the proposed KFAR model are the segments of the input images $A$ and $B$ that signify the forest and the non-forest segments of the image. The segments of the images are denoted as,

$$
S^{w}=\left\{S_{1}, S_{2}\right\}
$$

Where, $S_{1}$ and $S_{2}$ are the centroids signifying the segments denotes the forest and non-forest segments and $w \in\{A, B\}$ denoting the segments of the images $A$ and $B$. In other words, the prediction of the changes in the forest area of the instant $t$ is made through the satellite image of the instant $(t-1)$ and $t$ through extraction of the features and segmenting them using the automatic segmentation model, KFAR.

\section{E. Mapping the segments based on the pixel-wise Bhattacharya distance}

After segmenting the images, $A$ and $B$ as forest and non-forest regions, it is essential to map the segments of $A$ with respect to $B$ so that to identify the forest regions of $A$ in $B$ to discover the changes in the forest regions, which is done using the Bhattacharya distance. The region mapping is performed for individual pixels based on the minimal value of the Bhattacharya distance, which is formulated as,

$$
D(A, B)=\frac{1}{4} \ln \left[\frac{1}{4}\left(\frac{\sigma^{2} A}{\sigma^{2} B}+\frac{\sigma^{2} B}{\sigma^{2} A}+2\right)+\frac{1}{4}\left(\frac{\left(\mu_{A}+\mu_{B}\right)^{2}}{\sigma^{2} B+\sigma^{2} A}\right)\right]
$$


Where, $D(A, B)$ refers to the Bhattacharya distance between $A$ and $B$ distributions or segments. $\sigma^{2} A$ and $\sigma^{2} B$ corresponds to the variance of $A^{\text {th }}$ and $B^{\text {th }}$ distributions, and $\mu_{A}$ and $\mu_{B}$ are the mean of the $A^{\text {th }}$ and $B^{\text {th }}$ distributions.

\section{F. Forest change detection using the Pixel-based approach:}

Once the mapping is completed based on the pixel-wise Bhattacharya distance, the change in the forest area is determined. The change in the forest area for the current instance $t$ is based on the forest area of the instance $(t-1)$ for which initially, the intersection points $(A \cap B)$ is computed. Once the intersection points are derived, the intersection points are subtracted from the image $B$, which represent the satellite image of instant $t$.

\section{RESULTS AND DISCUSSION}

The section demonstrates the results of the proposed KFAR model in comparison with the comparative methods, and the analysis is made using the reference image of the years 2007. and 2008, respectively. The analysis based on the performance metrics enables the declaration of the effective method.

\section{A. Experimental setup}

The methods for forest change detection are implemented in MATLAB using the dataset taken from [24]. From the dataset two images are considered corresponding to both the years 2007 and 2008 in order to detect the changes in the forest area for the successive years, 2009, 2010, 2015, 2016, and 2017. The reference image_1 corresponds to the satellite image of 2007 taken from dataset with the north latitude 13 and east longitude 075, whereas the reference image_2 is obtained from the satellite image of the year 2008 taken from the dataset with the north latitude 13 and east longitude 075 . Similarly, the reference image_3 and reference image_4 are the satellite images of the year 2007 and 2008 with the north latitude 12 and east longitude 076.

\section{B. Experimental Results}

This section demonstrates the experimental results of the forest change detection using KFAR model using the images obtained from the dataset, Global PALSAR-2/PALSAR/JERS-1 with the Mosaic and Forest / Non-forest Maps. Figure 1 shows the sample results of the experiment and figure $1 \mathrm{a}$ ) and figure $1 \mathrm{~b}$ ) shows the original image of the year 2007 taken from database_1. The forest change results are enumerated in figure 1c).

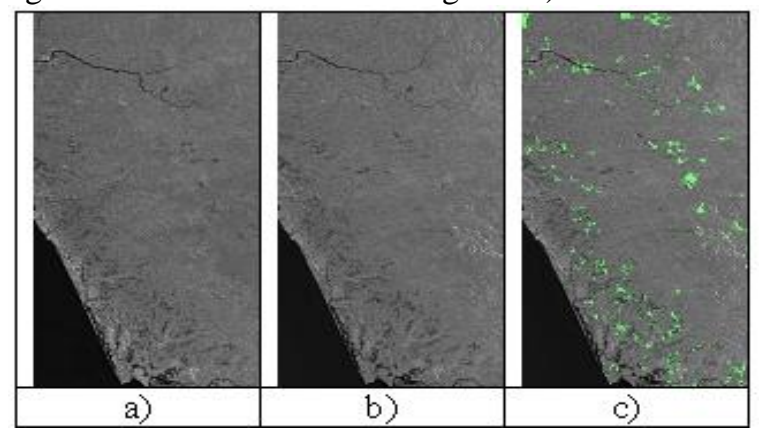

Figure 1. Sample results of the experiment using reference image (2007) of database 1, a) Original image (2015) from database_1, b) Original image (2015) from database_1, c) Forest change reported in 2007. Figure 2 shows the sample results of the experiment and figure 2 a) and figure 2 b) shows the original image of the year 2007 taken from database_ 2 . The forest change results are enumerated in figure $2 \mathrm{c}$ )

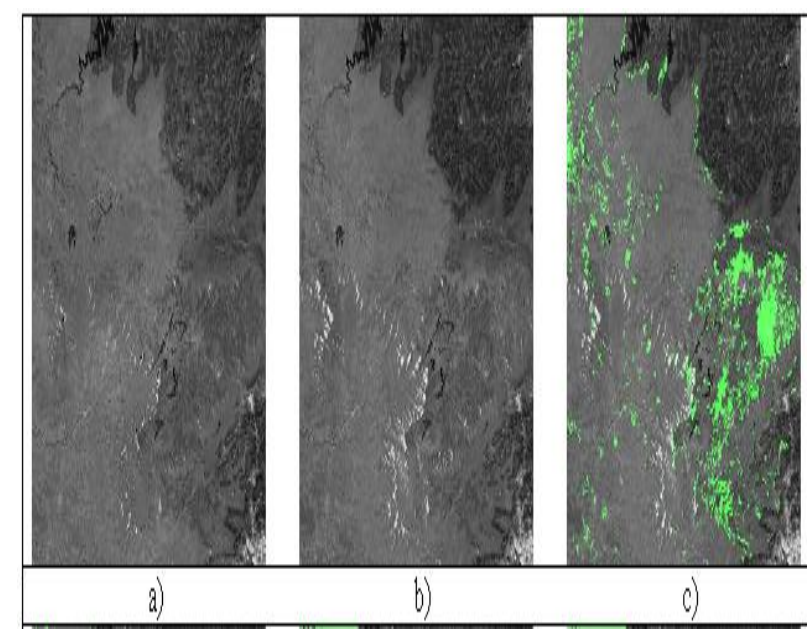

Figure 2. Sample results of the experiment using reference image (2007) of database 2, a) Original image (2015) from database_2, b) Original image (2015) from database_2, c) Forest change reported in 2007.

\section{CONCLUSION}

The forest change detection is performed using the proposed approach based on the pixel-based Bhattacharya distance for which the satellite images of two years are employed so that the change in the forest area with respect to the previous year is determined. To initiate the effective forest change detection with the satellite images, the vegetation indices that represent the feature vectors is extracted from the reference images after pre-processing using the bi-lateral filtering approach. The features are fed to the proposed segmentation method, Kernel Fuzzy Auto regressive model that is the integration of the Kernel Fuzzy C-Means Clustering and auto regressive model. The segments derived using the features are subjected to the mapping based on the pixel-based Bhattacharya distance and finally, the change in the forest is determined effectively using the pixel-based approach. The experimentation of the method is performed using two images taken from Global PALSAR-2 from 2007 and 2008. The analysis of the methods reveals that the proposed method acquired the minimal MSE and maximal accuracy of 0.0581 and 0.9211 . The future direction of the forest change detection is based on any optimization methods that would enhance the performance of the detection process.

\section{REFERENCES}

1. Manabu Watanabe, Christian N. Koyama, Masato Hayashi,Izumi Nagatani, and Masanobu Shimada,"Early-Stage

Deforestation Detection in the Tropics WithL-band SAR," IEEE

Journal of Selected Topics in Applied Earth Observations and Remote Sensing, vol. 11, no. 6, pp. 1-7, June 2018.

2. Unmesh Khati, Vineet Kumar, Debmita Bandyopadhyay, M Musthafa, and Gulab Singh, "Identification of forest cutting in managed forest of Haldwani, India using ALOS-2/PALSAR-2 SAR data," Journal of Environmental Management, vol. 213, pp. 503-512, May 2018. 
3. Akansha Singh and Krishna Kant Singh, "Unsupervised change detection in remote sensing images using fusion of spectral and statistical indices," The Egyptian Journal of Remote Sensing and Space Science, Available online 7 February 2018

4. Salman H. Khan, Xuming He, Fatih Porikli, and Mohammed Bennamoun, "Forest Change Detection in Incomplete Satellite Images With Deep Neural Networks," IEEE Transactions on Geoscience and Remote Sensing, vol. 55, no. 9, pp. 5407 - 5423, September 2017.

5. Neha Gupta, Gargi V. Pillai, and Samit Ari, "Change Detection in Optical Satellite Images Based on Local Binary Similarity Pattern Technique," IEEE Geoscience and Remote Sensing Letters, vol. 15, no. 3, pp. 389 - 393, March 2018

6. Hichem Sahbi, "Interactive Satellite Image Change Detection With Context-Aware Canonical Correlation Analysis," IEEE Geoscience and Remote Sensing Letters, vol. 14, no. 5, pp. 607 - 611, May 2017.

7. Oliver Cartus, Paul Siqueira, and Josef Kellndorfer, "An Error Model for Mapping Forest Cover and Forest Cover Change Using L-Band SAR," IEEE Geoscience and Remote Sensing Letters, vol. 15, no. 1, pp. 107 - 111, January 2018.

8. P.K.E. Pellikka, B.J.F. Clark, T. Sirviö and K. Masalin "Environmental change monitoring applying satellite and airborne remote sensing data in the Taita Hills, Kenya", Department of Geography, University of Helsinki, P.O. Box 64, 00014 Helsinki, Finland

9. Baudouin Desclée, Patrick Bogaert, and Pierre Defourny, "Forest change detection by statistical object-based method," Remote Sensing of Environment, vol. 102, pp.1-11, 2006.

10. R.Naveena Devi and G.Wiselin Jiji, "Change Detection Techniques - A Survey," International Journal on Computational Science \& Applications (IJCSA), Vol.5, No.2, April 2015.

11. T. Wang, G. T. Chen, H. L. Zhao, Z.-B. Dong, X.-Y. Zhang, X.-J Zheng, and N.-A. Wang, "Research progress on aeolian desertification process and controlling in north of China," J. Desert Res., vol. 26, no. 4, pp. 507- 516, Jul. 2006.

12. D. Sun, R. Dawson, H. Li, R. Wei, and B. Li, "A landscape connectivity index for assessing desertification: A case study of Minqin County, China," Landscape Ecol., vol. 22, no. 4, pp. 531-543, Apr. 2007.

13. R.Jafari, M.M.Lewis, and B.Ostendorf, "Animage-based diversity index for assessing land degradation in an arid environment in South Australia," J. Arid Environ., vol. 72, no. 7, pp. 1282-1293, Jul. 2008.

14. Geertam, S., \& Stillwell, "Planning Support Systems: Best practice and New Methods," New York. Springer, 2009.

15. Walter, V., Fritsch, D., "Automatic verification of GIS data using high resolution multispectral data," International Archives of Photogrammetry and Remote Sensing XXXII (Part 3/1), 485-489, 2000.

16. De Wasseige, C., and Defourny, P., "Remote sensing of selective logging impact for tropical forest management," Forest Ecology and Management, vol.188, pp. 161-173, 2004

17. Foody, G. M., "Remote sensing of tropical forest environments: Towards the monitoring of environmental resources for sustainable development," International Journal of Remote Sensing, vol. 24, pp. 4035-4046, 2003.

18. Singh, A., "Digital change detection techniques using remotely-sensed data," International Journal of Remote Sensing, vol. 6, pp. 989-1003, 1989.

19. Coppin, P., Jonckheere, I., Nackaerts, K., Muys, B., and Lambin, E., "Review article digital change detection methods in ecosystem monitoring: a review," International Journal of Remote Sensing, vol. 25, pp. 1565-1596, 2004.

20. Jensen, J.R., "Urban/Suburban Land Use Analysis, Manual of Remote Sensing," American Society of Photogrammetry, Falls Church, Virginia, pp. 1571-1666, 1983

21. Yi Ding, and Xian Fu, "Kernel-based fuzzy c-means clustering algorithm based on genetic algorithm", Neurocomputing, vol.188, pp.233-238, May 2016

22. Robert F. ENGLE and Simone MANGANELLI, "CAViaR Conditional Autoregressive Value at Risk by Regression Quantiles," American Statistical Association Journal of Business \& Economic Statistics, Vol. 22, No. 4, October 2004.

23. AndrésViña, Anatoly A.Gitelson, Anthony L.Nguy-Robertson, and Y Peng, "Comparison of different vegetation indices for the remote assessment of green leaf area index of crops", Remote Sensing of Environment, vol.115, no.12, pp.3468-3478, 15 December 2011.

24. Global PALSAR-2/PALSAR/JERS-1 Mosaic and Forest / Non-forest Map, "http://www.eorc.jaxa.jp/ALOS/en/palsar fnf/data/index.htm", accessed on September 2018.
25. GANESH B. SHINDE and VIJAYA B.MUSANDE, "Cotton Crop Classification method Using Levenberg-Marquardt Neural Network and its Performance Analysis on Vegetation and Green Leaf Indices", International Journal of Innovative Technology and Research (IJITR), vol.3, no.1, pp.1851 - 1857, December - January 2015.

26. Tri Muji Susantoro, Ketut Wikantika, Asep Saepuloh, and Agus Handoyo Harsolumakso, "Selection of vegetation indices for mapping the sugarcane condition around the oil and gas field of North West Java Basin, Indonesia", In Proceedings of the IOP Conference Series: Earth and Environmental Science, vol.149, no.1, May 2018.

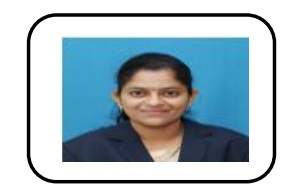

\section{AUTHORS PROFILE}

Ms. Madhuri B. Mulik, Currently Pursuing PHD from Visvesvaraya Technological University, Belgavi. She has completed her Master in Technology in Electronic Technology in 2011 from Department of Technology in Shivaji University, Kolhapur. Also completed one year Post Graduation Diploma in GIS. She has expertise in Image processing, Embedded System, VLSI Design. Currently working as a Assistant Professor in Electronics \& Telecommunication Engineering in Sharad Institute of Technology College of Engineering, Yadrav.

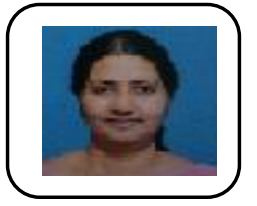

Dr. (Mrs). V. Jayashree, Professor HOD Electrica Engineering \& PG Coordinator (Electronics Engineering), DKTE Society's Textile and Engineering Institute, Ichalkarnji, Maharashtra. Completed Master in Engineering \& PHD from Shivaji University, Kolhapur. She holds a Second Rank in Bachelor in Engineering in Electrical Engineering from Karnataka University, Dharwad. Expertise areas are Image Processing \& Pattern Recognition, Artificial Intelligence, VLSI Design, and Microcontroller.

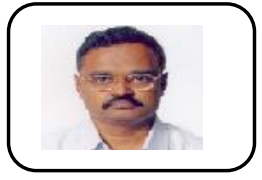

Dr. Pandurangarao N. Kulkarni, Professor and Head Department of Electronics \& Communication Engg. Basaveshwar Engineering College, Bagalkot, Karnataka State, India. Received PHD from Indian Institute of Technology, Bombay in 2010 in Speech Processing. He received Master in Technology in Advanced Communication National Institute of Technology Suratkal, Karnataka state, India in 1995 B.E Electronics \& Communication Engineering. Karnataka University, Dharawad in 1988. Areas of interest are Speech signal processing for improving speech perception, Digital Signal Processing and applications, Digital communication. 